\title{
El liderazgo docente en el marco de la emergencia sanitaria
}

\author{
Carlos Jorge Santisteban Llonto \\ Educarlos67@yahoo.es \\ Orcid: 0000-0001-5817-4749 \\ Instituto Superior Pedagógico Privado Don Bosco \\ Chacas-Perú
}

\section{RESUMEN}

El docente es considerado uno de los factores más importantes en la educación, para lograr los resultados en los aprendizajes, de allí la importancia del liderazgo docente en la educación a distancia o virtual en el contexto pandémico, para lograr las metas institucionales respecto a las competencias propuestas en el currículo, con la aplicación de estrategias didácticas dinámicas, mediadas por el poder de la tecnología. En tal sentido el propósito fue analizar el liderazgo docente en el marco de la emergencia sanitaria, para ello se inició la indagación de la información en los motores de búsqueda: Scielo, Redalyc, Dialnet, Ebesco utilizando los filtros respectivos para acotar el conocimiento, así mismo se utilizó fichas de trabajo, para el procesamiento de la información y el software Mendeley, para clasificar, ordenar y citar a los autores estudiados. Respecto a los resultados el liderazgo docente impacta en los aprendizajes y en la formación integral del estudiantado con estrategias activas como el aprendizaje basado en problemas (ABP) y mediados por las tecnologías de la comunicación e información (Tics). El liderazgo docente ha respondido al contexto actual con creatividad, resiliencia y habilidades tecnológicas para sacar adelante a la educación.

Palabras clave: estrategias; liderazgo docente, liderazgo instruccional, liderazgo transformacional; logros de aprendizaje. 


\title{
Teaching leadership in the framework of the health emergency
}

\begin{abstract}
The teacher is considered one of the most important factors in education, to achieve the results in learning, hence the importance of teacher leadership in distance or virtual education in the pandemic context, to achieve institutional goals regarding the competences proposed in the curriculum, with the application of dynamic didactic strategies mediated by the power of technology. In this sense, the purpose was to analyze the teaching leadership in the framework of the health emergency, for this the investigation of the information in the search engines began: Scielo, Redalyc, Dialnet, Ebesco using the respective filters to limit the knowledge, thus The same worksheets were used to process the information and the Mendeley software to classify, order and cite the authors studied. Regarding the results, the teaching leadership impacts on the learning and the integral formation of the student body with active strategies such as problembased learning (PBL) and mediated by information and communication technologies (ICT). Teacher leadership has responded to the current context with creativity, resilience, and technological skills to move education forward.
\end{abstract}

Keywords: strategies; teacher leadership, instructional leadership, transformational leadership; learning achievements.

Artículo recibido: 10 Agosto. 2021 Aceptado para publicación: 07. Setiembre. 2021

Correspondencia: Educarlos67@yahoo.es Conflictos de Interés: Ninguna que declarar 


\section{INTRODUCCIÓN}

El liderazgo educativo es un tema de interés y de importancia, para la comunidad educativa. Al respecto Maureira, Garay y Lopez (2016), Morales,Torres y Rendon (2018), refieren que el liderazgo directivo crea las condiciones necesarias, para el logro de los aprendizajes de los adolescentes. En esta misma línea Rodríguez \& López (2020) manifiestan que, los directivos favorecen las condiciones para el aprendizaje con metodologías basadas en proyectos. Por su parte Casas, (2019), relata que, el liderazgo pedagógico tiene como centro el aprendizaje de los jóvenes, siendo el motor de los establecimientos educativos.

El Liderazgo docente de acuerdo a Lin et al (2020) y Shen, (2020) revelan que, el aprendizaje de los estudiantes, en el área de matemática se relacionan con el liderazgo del docente en el aula, la relación fuerte se da en las interacciones del docente y los enfoques de la enseñanza. Otro trabajo de Ilma \& Suyatno (2020) resuelven que, el liderazgo docente afecta las necesidades motivacionales de los escolares y por ende los resultados del aprender. Para Gonzales, Palomares, López, Gento. (2019) refieren que la formación continua del docente es clave, para ejercer la práctica del liderazgo y dar respuesta a los retos y desafíos que plantea y exige la sociedad actual.

Pero ¿Qué es liderazgo docente? Bush \& Glover (2003), Leithwood (2009) y (Bolivar, 2017), los autores en mención coinciden que el liderazgo es un proceso de influencia, concepto que está arraigado actualmente en el mundo de la educación. Otra idea de Vásquez, Bernal y Orús, (2014), refieren que el liderazgo es una capacidad para dirigir. Teniendo en cuenta a los autores señalados y para efectos del presente trabajo, se puede delimitar el concepto de liderazgo como la capacidad y habilidad del docente, para influir en los estudiantes en el logro de los aprendizajes y en su formación integral. Desde esta mirada, se aborda el tema liderazgo docente en situaciones de crisis sanitaria mundial.

La COVID-19 ha impactado en los Sistemas Educativos de todo el mundo. Por ejemplo, en el Perú, según la UNESCO (2020), 9 millones de estudiantes son afectados aproximadamente por el cierre de las escuelas en todo el territorio nacional, lo que obliga a transitar de una educación presencial a una educación a distancia (Ministerio de Educación del Perú-MINEDU, 2020), en este contexto el docente tiene que liderar el aprendizaje y lograr las competencias previstas en el diseño Curricular, a pesar que en la zona rural solo el 5,8\% y 40,1\% a nivel nacional de los hogares tienen acceso al internet 
y al uso de recursos tecnológicos como los celulares, laptop y computadoras (Instituto Nacional de Estadística Del Perú, 2020).

El liderazgo docente se analiza desde los enfoques teóricos del liderazgo instruccional y transformacional, porque ejercen una influencia en el desarrollo de las competencias de los estudiantes; en los resultados de los aprendizajes, en la mejora del aprender a través de estrategias participativas y dinámicas. Cada una de las teorías están enmarcada en el contexto educativo y en relación directa al accionar pedagógico del docente.

El liderazgo docente también se relaciona con las teorías de Ausubel, Bruner y Vygotsky, porque consideran que el aprendizaje debe ser significativo y retador, así mismo esté vinculado a la realidad social, para resolver las problemáticas y necesidades de la comunidad. En este sentido el liderazgo docente tiene el rol de guía y mediador de las experiencias del aprendizaje, partiendo de lo que saben los estudiantes, pero puede llevarlos a nuevos conocimientos con su mediación y liderazgo. Experiencias que se desarrollan en la educación a distancia y virtual por efecto de la emergencia sanitaria. El tema del liderazgo tiene un interés en diversos campos del conocimiento, pero de modo particular en el ámbito educativo, de allí nace la motivación de realizar la investigación cuyo objetivo general es analizar el liderazgo docente en el contexto de la emergencia sanitaria, siendo los objetivos específicos: a)describir el liderazgo docente en el contexto de la emergencia sanitaria b)identificar las estrategias del liderazgo docente en el contexto de la pandemia c) explicar la importancia del liderazgo docente en la emergencia sanitaria

\section{METODOLOGIA}

Un artículo de revisión es el estudio selectivo y crítico de un tema específico y de interés. Tiene como propósito analizar bibliografía publicada y situarla en una perspectiva.(Vera, 2017,Merino, 2013,Reyes, 2020). En tal sentido se ha realizado una revisión selectiva de material bibliográfico publicado en revistas indexadas, artículos científicos y de revisión con el fin de realizar un análisis, crítico y reflexivo de la información.

La búsqueda de los documentos se realizó en la base de datos Ebesco, Scielo, Dialnet y Redalyc entre el periodo de agosto del año 2020 a marzo del 2021, a través de los términos de búsqueda: liderazgo docente, liderazgo educativo, liderazgo instructivo, liderazgo transformacional, estrategias didácticas del docente. Para identificar artículos relevantes y actuales se tuvo en cuenta solo el periodo entre los años 2016 al 2021 respectivamente, 
en razón a ello se consideró 60 documentos relacionados al tema de estudio mediante la lectura del título, resumen, palabras clave, resultados y conclusiones, quedando seleccionados 30 estudios, para este proceso se ha utilizado el software Mendeley, el cual permitió la organización de las referencias bibliográficas.

Los filtros empleados fueron: idioma y tipos de publicación. Con la finalidad de acotar el número se introdujo el filtro idioma, es decir aquellos que están publicados en inglés y español. Otro criterio fue el área de investigación, considerando solo al área de educación. Así mismo, el criterio de documentos de acceso abiertos. Finalmente, para el análisis de la información y la síntesis respectiva se utilizó una plantilla con los datos enfocados en las variables de interés del estudio: a) autor b) nombre de revista indexada c) año d) cita textual e) parafraseo y d) referencia bibliográfica.

Plantilla de análisis y síntesis de la información

\begin{tabular}{|l|c|l|l|l|l|}
\hline Autor & $\begin{array}{c}\text { Revista } \\
\text { indexada }\end{array}$ & Año & Cita textual/resumen & Parafraseo & $\begin{array}{c}\text { Referencia } \\
\text { bibliográfica }\end{array}$ \\
\hline & & & & & \\
\hline
\end{tabular}

Fuente: Elaboración propia

\section{RESULTADOS}

\section{Liderazgo docente}

El liderazgo Docente es la capacidad para influir en el logro de los aprendizajes, y en el desarrollo integral de los estudiantes en la educación a distancia y virtual, por el efecto de la COVID-19. Sin embargo, en la literatura revisada apenas se le menciona y lo relacionan a la actualización y al desarrollo profesional.(Arbués \& García, 2014). El liderazgo docente en el contexto pandémico es un factor central en la comunidad educativa, porque moviliza las competencias del estudiantado, anima y motiva el proceso del aprendizaje con retos y desafíos es decir el docente influye de manera positiva en el logro de la metas institucionales y en la mejora de la calidad educativa.(Palacios et al., 2020).

El liderazgo docente se sitúa en dos enfoques: liderazgo instruccional, y transformacional.

En Primer lugar, el liderazgo instruccional o pedagógico explica que el docente se enfoca en la planificación curricular, enseñanza aprendizaje, evaluación, desarrollo profesional y los procesos de mejora. ( Martínez \& Ibarrola 2015;Gajardo \& Ulloa, 2016; Sepúlveda \& Volante, 2019). Desde este horizonte, el docente de manera colegiada, planifica 
proyectos de aprendizaje desafiantes y retadores, también se apoya en las tecnologías, para integrar plataformas con metodologías activas, para desarrollar el aprendizaje a distancia y virtual en el contexto de la pandemia y lograr resultados en el aprendizaje (Cheng, 2021).

El liderazgo instruccional del docente influye de manera directa en el logro de los aprendizajes del estudiantado, porque preparan las experiencias del aprender planteando problemas de la realidad, para su solución, lo ejecutan y evalúan. Los directivos también influyen de manera indirecta creando las condiciones necesarias, para el aprendizaje; en tal sentido, ambos liderazgos se complementan y tienen como base el liderazgo instruccional. (Robinson, Lloyd, Rome., 2014; Balduzzi, 2015; Pàmies Rovira et al., 2016; Aravena, Cadiz, Peña,Gonzales y Nuñez, 2019).

En segundo lugar, tenemos al liderazgo transformacional que se sitúa en el contexto del ámbito educativo y está asociado a la idea de cambio, a los efectos de las practicas transformacionales respecto al aprendizaje y a la comunidad educativa. Siguiendo a Leithwood (2009), y en concordancia con Robinson (2007), refieren que el modelo está configurado por las siguientes dimensiones: dirección de futuro, es decir tener una visión compartida y metas claras; desarrollo profesional: brindando atención a las necesidades y fortalecimiento de sus capacidades; rediseñar a la organización, ello quiere decir : horarios, normas, relaciones cordiales, conexión de la escuela con el entorno y oportunidades, así como una cultura de colaboración; gestión del aprendizaje-enseñanza: planificación, mediación y evaluación, monitoreo y acompañamiento.

El liderazgo transformacional, se relaciona con el liderazgo pedagógico, respecto a la dimensión de la gestión del aprendizaje, pero también con los otros aspectos, es decir ambos liderazgos se complementan, para garantizar los aprendizajes de los estudiantes en el contexto pandémico.(Altamirano., Carranza y Pila, 2021;Kochen,2020). Desde esta mirada, el docente realiza los cambios pertinentes en las actividades de aprendizaje propuestas, materiales y recursos, estrategias y la evaluación, para responder a las necesidades y demandas del estudiantado en la educación virtual y a distancia.

El modelo transformacional motiva e inspira al docente, a la reflexión sobre: el logro de las competencias, enfoques, estándares de calidad, actividades de aprendizaje, estrategias, entornos virtuales, equipos tecnológicos y la evaluación, es decir de la gestión curricular, para tomar decisiones y compromisos que conlleven a mejorar la práctica pedagógica, los 
proyectos de aprendizaje, las estrategias didácticas y los resultados del aprendizaje. En este sentido el docente se convierte en un pilar de la educación, porque se alinea a la mejora continua del aprendizaje en la educación virtual y a distancia. (Rafael et al., 2020; Ojeda \& Palacios, 2021). Este tipo de respuesta de parte del profesorado son favorables, para los estudiantes y la comunidad educativa, por ende, a la educación.

\section{Estrategias del liderazgo docente}

La pandemia ha generado cambios en la vida social, cultural, económica y en la educación. Todas las escuelas y universidades del mundo fueron cerradas, para evitar el contagio de la Covid-19. En razón a ello, se pasó de una educación presencial a una educación virtual y a distancia, para garantizar el derecho a la educación de los estudiantes. En este contexto conviene formularnos las siguientes preguntas desde la mirada del liderazgo pedagógico, transformacional y conectivista de Siemens: ¿Qué y cómo aprenden los estudiantes? ¿Con qué recursos aprenden? y ¿Cómo son evaluados?

Solórzano \& García (2016), refieren que el conocimiento está en la red y el aprendizaje consiste en la habilidad de construir y navegar en las redes. Según Siemens \& Fonseca, (2007) el aprendizaje y el conocimiento dependen de la diversidad de opiniones, es un proceso de conectar nodos y reside en dispositivos. Desde esta mirada el rol del docente es de guía, líder, mediador, acompañante y estimulador del aprendizaje (Cueva, Garcia, Martinez, 2020; Islas , 2021). En razón a ello, el docente debe diseñar un conjunto de estrategias, para desarrollar el aprendizaje virtual y a distancia.

Se entiende por estrategia al conjunto de procedimientos y recursos que utiliza el maestro, para el desarrollo de aprendizajes significativos; otra idea, son los distintos pasos que conllevan al aprendizaje basado en retos y desafíos con la finalidad de desarrollar competencias en los estudiantes.(Diaz, 1998; Tébar, 2003). Por tal motivo, las estrategias deben ser funcionales, útiles, prácticas, vivenciales, significativas y mediadas con las tecnologías de la información y comunicación en adelante (Tics).

Las estrategias didácticas que vienen aplicando los docentes, para desarrollar el aprendizaje, en el contexto pandémico, están sustentadas por los tics. En este sentido, uno de los recursos, para desarrollar las actividades de aprendizaje es el Google meet o zoom, donde se realizan presentaciones con diapositivas las situaciones problemáticas, actividades de aprendizaje, productos y evaluación.(Vialart,2020).Otro medio que resulta muy útil, para desarrollar las actividades de aprendizaje, la comunicación, y 
retroalimentación es el WhatsApp.(Sapién et al., 2020; Salle \& Madrid, 2021). Los medios señalados permiten la interacción entre docente y estudiante, para la recuperación de saberes previos, motivación constante, gestión del conocimiento y la evaluación formativa frente a otras herramientas como los wikis, blogs o las redes sociales.

Otra de las estrategias para fomentar el aprendizaje colaborativo entre los estudiantes es la herramienta del Google drive, permitiendo un trabajo sincrónico y asincrónico, así mismo permite el seguimiento a cada uno de los equipos de trabajo (Gabriela et al, 2021; Cotán Fernández et al., 2021). De ello, se infiere que los docentes han usado diversos recursos que ofrece Google como elementos estratégicos para desarrollar aprendizajes significativos, entre los que podemos mencionar: Google classroom, es un aula virtual, para el desarrollo de los aprendizajes, los formularios permiten realizar encuestas, evaluar los procesos del aprender y otras necesidades. (González, 2021).

La estrategia del aprendizaje basado en problemas en adelante ABP, es clave para desarrollar capacidades, está centrada en el estudiante, proponen retos y desafíos, es motivadora y dinámica, es decir tiene un efecto significativo y favorable en el resultado de los aprendizaje (Colón Ortiz \& Ortiz-Vega, 2020; Esther \& Medina, 2021; Hernandez, E., Yallico, 2020). En el contexto de la educación a distancia o virtual el ABP es una metodología necesaria para desarrollar el aprendizaje en conexiones y los estudiantes aprendan a resolver problemas del contexto

El ABP como estrategia de trabajo tiene una estructura y según el Ministerio de Educación del Perú, (MINEDU, 2013) propone los siguientes elementos: a) planificación, que consiste en: definición de una situación o problema como punto de partida y determinar el título del proyecto. b) implementación, es decir, investigar, indagar, realización de actividades o tareas individuales o colectivas, c) comunicación que implica socialización de los productos del proyecto, d) proceso de evaluación.

\section{Importancia del liderazgo docente en el contexto de la pandemia}

El liderazgo docente en la educación virtual y a distancia en el contexto pandémico, tiene una gran importancia porque, el docente acompaña el proceso del aprendizaje de los estudiantes, les ayuda a reflexionar a través de preguntas retadoras, para superar las dificultades, les plantea retos y desafíos problemáticos del contexto, así mismo retroalimenta el aprendizaje destacando los logros y sugerencias de mejora, la red por sí solo no guía, ni orienta; de allí la importancia del rol del liderazgo docente para lograr 
las competencias, capacidades y estándares de aprendizaje, expresados en resultados. (Blanco \& Amigo, 2016). Desde este horizonte el rol del liderazgo docente se convierte en un pilar fundamental de la educación en el contexto de la emergencia sanitaria, para la comunidad educativa y la sociedad.

El liderazgo docente es importante porque está ayudando a salvaguardar la salud física y emocional de los estudiantes y sus familias que se ha visto gravemente afectada por las medidas dadas, para prevenir el contagio de la COVID-19. Ofreciéndoles soporte emocional a través de diversas actividades como escuchar y cantar una canción juntos, comentar frases motivadoras, para mirar el mundo con esperanza y optimismo, de modo que alivie las angustias, temores y ansiedades a fin de restablecer el equilibrio en la familia y en el entorno socio cultural.

El liderazgo docente es importante porque le hace frente a la adversidad de la crisis sanitaria con resiliencia y habilidades tics para el desempeño del trabajo pedagógico.(Sanz Ponce, R. y López Luján, 2021). El despertar de nuevas habilidades, en situaciones de crisis, favorece la creatividad, innovación e investigación pedagógica para proponer proyectos de aprendizaje, diseños curriculares flexibles, aplicación de las tecnologías en los procesos pedagógicos con metodologías activas centradas en los estudiantes de acuerdo a sus necesidades e intereses, es decir el liderazgo es un elemento de transformación y mejora de la educación aun en tiempo difíciles.

\section{CONCLUSIONES}

El liderazgo docente en el contexto pandémico resulta fundamental porque está llevando adelante el aprendizaje de los estudiantes, es decir que se logren las competencias, capacidades y resultados esperados; para ello se apoya en estrategias didácticas y el poder de la tecnología.

1. El liderazgo docente influye en el logro de los aprendizajes y en el desarrollo integral de los estudiantes en la educación virtual y a distancia. Tienen como modelo al liderazgo pedagógico, porque esta enfocado en la enseñanza aprendizaje, así mismo el modelo transformacional, porque se realiza la mejora continua del aprendizaje y conectivista porque el conocimiento esta en las redes y el aprendizaje es la habilidad para construir el saber y navegar.

2. Las estrategias son un conjunto de pasos, procedimiento y recursos que aplica el líder docente, para desarrollar aprendizajes significativos, mediados por los tics en el 
contexto pandémico. Los medios más utilizados han sido el Google meet, whats App, Google drive, Google classroom y formularios. El ABP es una estrategia centrada en el estudiante, moviliza capacidades e influye de manera favorable en los resultados de los aprendizajes.

3. El liderazgo docente es importante porque responde creativamente a los retos y desafíos que le plantea la emergencia sanitaria con resiliencia, habilidades tics, pero sobre todo logrando las competencias y capacidades de los aprendizajes de los estudiantes.

\section{RECOMENDACIONES}

1. La comunidad educativa debe tomar conciencia del liderazgo docente para lograr resultados favorables en el aprendizaje de los estudiantes. Los docentes deben reflexionar en las oportunidades de mejora, para implementar proyectos innovadores en la mejora continua del aprendizaje.

2. Los resultados de los aprendizajes pueden mejorar con la implementación de recursos y estrategias innovadoras que movilicen las capacidades del estudiantado y del colegiado del liderazgo docente.

3. El liderazgo docente es clave para el logro de las competencias, capacidades y estándares de aprendizaje, por lo que es necesario el apoyo de los padres de familia y la sociedad civil.

\section{REFERENCIAS BIBLIOGRAFICAS}

Altamirano, E., Carrera, J. E., \& Pilar, J. (2021). Dirección y Profesorado: Factores Clave para el Liderazgo e Innovación en Tiempos de Pandemia. Revista TecnológicaEducativa Docentes 2.0, 10(1), 32-43. https://doi.org/10.37843/rted.v10i1.175

Aravena, F., Cádiz, M., Peña, C., González, M., \& Nuñez, C. (2019). Liderazgo escolar: Una mirada a los criterios de éxito pedagógico de los Jefes de Unidad Técnica Pedagógica (UTP) en Chile. Calidad En La Educación, 51, 252. https://doi.org/10.31619/caledu.n51.647

Arbués, E., \& Ibarrola, S. (2014). Leadership in Teachers: A Requirement for Employability. Procedia - Social and Behavioral Sciences, 139, 472-478. https://doi.org/10.1016/j.sbspro.2014.08.046

Balduzzi, E. (2015). Liderazgo educativo del profesor en el aula y la personalización educativa. Revista Española de Pedagogía, 73(260), 141-156. 
Bernal , A., \& Ibarrola , S. (2015). Liderazgo del profesor: objetivo básico de la gestión educativa. Revista Iberoamericana de Educación, 67, 55-70. https://doi.org/10.35362/rie670205

Blanco, A., \& Amigo, J. (2016). El rol del docente en la era digital. Revista Interuniversitaria de Formación Del Profesorado, 30(2), 103-114.

Bolivar, A. (2017). Liderazgo educativo y desarrollo profesional docente: Una revisión internacional.

February, 61-103. https://www.researchgate.net/profile/Antonio_Bolivar/publication/313793757_L iderazgo_educativo_y_desarrollo_profesional_docente_Una_revision_internacio nal/links/58a5fe3f92851cf0e3a6ac16/Liderazgo-educativo-y-desarrolloprofesional-docente-Una-revision-int

Bush, T., \& Glover, D. (2003). School Leadership : Concepts and Evidence. National College for School Leadership, Spring, 1-42.

Casas, A. (2019). Liderazgo pedagógico, nuevas perspectivas para el desempeño docente.

Cheng, J. N. (2021). Tecnologías de la Información y Comunicación en el Desarrollo de las Competencias Matemáticas en la Educación Virtual Universitaria Information and Communication Technologies in the Development of Mathematical Competences in Virtual University Education. 2215, 2908-2930.

Colón , L., \& Ortiz, J. (2020). Efecto del Uso de la Estrategia de Enseñanza Aprendizaje Basado en Problemas (ABP) en el Desarrollo de las Destrezas de Comprensión y Análisis de la Estadística Descriptiva. Revista Iberoamericana de Evaluación Educativa, 13(1), 205. https://doi.org/10.15366/riee2020.13.1.009

Cotán , A., García , I., \& Gallardo , A. (2021). Trabajo colaborativo en línea como estrategia de aprendizaje en entornos virtuales: una investigación con estudiantes universitarios de Educación Infantil y Educación Primaria. Educación, 30(58), 147-168. https://doi.org/10.18800/educacion.202101.007

Cueva, J., Garcia, A., Martinez, O. (2020). La influencia del conectivismo para el uso de las TIC en el proceso enseñanza-aprendizaje. 2017(1), 1-9.

Diaz, F. (1998). Una aportación a la didáctica de la historia. La enseñanza-aprendizaje de habilidades cognitivas en el bachillerato. Perfiles Educativos, núm. 82, octubre-di, 1998 Instituto de Investigaciones sobre la Universidad y la Educación 
Distrito Federal, México.

Esther, L., \& Medina, A. (2021). Oportunidades del Aprendizaje Basado en Proyectos $(A B P)$ para la pedagogía profesional en la Universidad de Holguín, Cuba.

Gabriela, A., Arroyo, C., David, O., \& Escobar, O. (2021). Uso de google drive como estrategia de enseñanza aprendizaje en asignaturas de investigación en alumnos de nutrición. Revista Educación y Tecnología, 23-46.

Gajardo, J., \& Ulloa, J. (2016). Liderazgo Pedagógico, Conceptos y Tensiones Nota Técnica $\mathrm{N}^{\circ}$ 6. Nota Técnica $N^{\circ}$ 6. LIDERES EDUCATIVOS, Centro de Liderazgo Para La Mejora Escolar: Universidad Concepción, Chile., 6, 13. https://www.lidereseducativos.cl/wp-content/uploads/2017/01/NT-6.pdf

Gonzales, R., Palomares, A., López, E., Gento, S. (2019). Exploring Teacher' S Pedagogical Leadership : the Formative Dimension. 24, 9-25.

González, A. (2021). Percepción estudiantil de un curso universitario que integró los recursos de Google como apoyo educativo. RIDE Revista Iberoamericana Para La Investigación y El Desarrollo Educativo, 11(22). https://doi.org/10.23913/ride.v11i22.962

Hernandez, E., Yallico, R. (2020). El Aprendizaje Basado en Problemas (ABP) como estrategia didáctica innovadora en la enseñanza de la Anatomía Humana.

Ilma , Z., \& Suyatno, S. (2020). The effect of teachers' leadership on students' motivation in al-Islam Tambakbayan elementary school. Universal Journal of Educational Research, 8(5), 1907-1915. https://doi.org/10.13189/ujer.2020.080527

Instituto Nacional de Estadística del Perú. (2020).el 41\% tuvo acceso a los recursos tecnológicos

Islas , C. (2021). Conectivismo y neuroeducación: transdisciplinas para la formación en la era digital. CIENCIA Ergo Sum, 28(1), 1-13. https://doi.org/10.30878/ces.v28n1a11

Kochen, G. (2020). La gestión directiva o el liderazgo educativo en tiempos de pandemia. Innovaciones Educativas, 22(33), 9-14. https://doi.org/10.22458/ie.v22i33.3349

Leithwood, K. (2009). ¿Cómo liderar nuestras escuelas? Aportes desde la investigación. www.fundacionchile.cl

Lin, W., Yin, H., Han, J., \& Han, J. (2020). Teacher-student interaction and chinese students' mathematics learning outcomes: The mediation of mathematics 
achievement emotions. International Journal of Environmental Research and Public Health, 17(13), 1-17. https://doi.org/10.3390/ijerph17134742

Maureira , Ó., Garay, S. \& López, P. (2016). Reconfigurando el sentido del liderazgo en organizaciones escolares contemporáneas: La perspectiva del liderazgo distribuido. Revista Complutense de Educacion, 27(2), 689-706. https://doi.org/10.5209/rev_RCED.2016.v27.n2.47079

Merino, A. (2013). Como escribir documentos científicos. Artículo de revisión. Salud En Tabasco, 19(3), 90-94.

MINEDU. (2013). Los proyectos de aprendizaje para el logro de competencias Educación primaria. 124. www.minedu.gob.pe

MINEDU. (2020). Resolución Ministerial 160-2020-MINEDU. Diario El Peruano, 15, 2. https://cdn.www.gob.pe/uploads/document/file/574684/disponen-el-inicio-delano-escolar-a-traves-de-la-implementa-resolucion-ministerial-n-160-2020minedu-1865282-1.pdf

Morales, I., Torres, B y Rendon, A. (2018). Liderazgo educativo de la universidad de Guayaquil. 15(2), 2017-2019. https://www.uam.es/gruposinv/meva/publicaciones jesus/capitulos_espanyol_jesus/2005_motivacion para el aprendizaje Perspectiva alumnos.pdf\%0Ahttps://www.researchgate.net/profile/Juan_Aparicio7/publicati on/253571379_Los_estudios_sobre_el_cambio_conceptual_

Ojeda, N., \& Palacios, Y. (2021). Relación intrínseca del liderazgo, tecnologías, COVID y educación, un espacio de reflexión para situaciones pandémicas. Relación Intrínseca Del Liderazgo, Tecnologías, COVID y Educación, Un Espacio de Reflexión Para Situaciones Pandémicas, 45, 96-122. https://eds-a-ebscohostcom.bibliotecavirtual.udla.edu.ec/eds/detail/detail?vid=0\&sid=f839d6e5-f1ee4213-b4f1e2f4a7182ab3\%40sessionmgr4007\&bdata=Jmxhbmc9ZXMmc210ZT11ZHMtbG 12ZQ\%3D\%3D\#AN=149212353\&db=fua

Palacios, G., González, R., \& Sención, H.(2020). Affective dimension of teacher's pedagogical leadership. Revista Complutense de Educacion, 31(4), 485-495. https://doi.org/10.5209/rced.65635

Pàmies , J., Senent ,J.\& Essomba , M. (2016). El liderazgo pedagógico y la implicación 
del profesorado como factores de éxito en centros de entornos desfavorecidos en España. RELIEVE - Revista Electrónica de Investigación y Evaluación Educativa, 22(2), 1-15. https://doi.org/10.7203/relieve.22.2.7600

Rafael, M., Fernández, V., \& Sevilla, P. (2020). El profesorado como líder en situaciones de crisis : el caso de la pandemia por COVID-19. 81-91.

Reyes, H. (2020). Artículos de Revisión. Revista Medica;148:103-108, 103-108. http://www.inafed.gob.mx/work/enciclopedia/EMM14jalisco/municipios/14067a .html

Robinson, V., Lloyd, C., \& Rowe, K. (2014). El impacto del liderazgo en los resultados de los estudiantes: un análisis de los efectos diferenciales de los tipos de liderazgo. REICE: Revista Electrónica Iberoamericana Sobre Calidad, Eficacia y Cambio En Educación, 12(4), 13-40.

Robinson, V. (2007). School Leaderschip and Student Outcomes: Identifying What Works and Why. October, 41, 1-32.

Rodríguezo, M. \& López, R. (2020). La dirección escolar : Liderazgo pedagógico y mejora escolar School management: Pedagogical leadership and school improvement. 38, 275-292.

Salle, L., \& Madrid, C. (2021). Herramientas que facilitan el aprendizaje colaborativo en entornos virtuales: nuevas oportunidades para el desarrollo de las ecologías digitales de aprendizaje. 39, 81-100.

Sanz , R. y López , E. (2021). (2021). Consecuencias pedagógicas entre el alumnado de enseñanza básica derivadas de la Covid-19. Una reflexión en torno a los grandes $\begin{array}{llll}\text { olvidados de la } & \text { 149-166. }\end{array}$ https://doi.org/https://doi.org/10.14201/teri.25471

Sapién , A., Piñón , L., Gutiérrez , M, \& Bordas , J. (2020). La Educación superior durante la contingencia sanitaria COVID-19: Uso de las TIC como herramientas de aprendizaje. Caso de estudio: alumnos de la Facultad de Contaduría y Administración. Revista Latina de Comunicacion Social, 2020(78), 309-328. https://doi.org/10.4185/RLCS-2020-1479

Sepúlveda, R., \& Volante, P. (2019). Instructional middle leadership: International Approaches for professional development in Chilean schools. Profesorado, 23(3), 341-362. https://doi.org/10.30827/profesorado.v23i3.11231 
Siemens, G., \& Fonseca, D. (2007). Conectivismo. 2004, 1-11.

Solórzano, F., \& García, A. (2016). Fundamentos del aprendizaje en red desde el conectivismo y la teoría de la actividad. Revista Cubana de Educación Superior, 35(3), 98-112. http://scielo.sld.cu/scielo.php?script=sci_abstract\&pid=S025743142016000300008

Tébar, L. (2003). El perfil del profesor mediador. Aula XXI, Santillana, Madrid. Aula XXI, Santillana, Madrid, 2009.

UNESCO. (2020). El sistema educativo peruano: buscando la calidad y la equidad durante los tiempos de COVID-19.

Vásquez, S., Bernal, J y Orús, M. (2014). La conceptualización del liderazgo : una aproximación desde la práctica educativa Leadership conceptualization: an approach from the educational practice Introducción. Revista Iberoamericana Sobre Calidad, Eicacia y Cambio En Educación, 12(5), 79-97. https://repositorio.uam.es/bitstream/handle/10486/663351/REICE_ 5_6.pdf?sequence $=1 \&$ is Allowed $=\mathrm{y}$

Vera, O. (2017). Artículos de Revisión. Revista Chilena de Ortopedia y Traumatología, 58(02), 033-033. https://doi.org/10.1055/s-0037-1606585

Vialart , M (2020). Estrategias didácticas para la virtualización del proceso enseñanza aprendizaje en tiempos de COVID-19. Educación Médica Superior, 34(3). 